\title{
Gradhiva
}

GRADHI

Revue d'anthropologie et d'histoire des arts

11 | 2010

Grands hommes vus d'en bas

\section{Brigitte Derlon et Monique Jeudy-Ballini, La Passion de l'art primitif. Enquête sur les collectionneurs}

Paris, Gallimard, coll. «Bibliothèque des sciences humaines », 2008, $322 \mathrm{p}$.

Nathalie Heinich

\section{(2) OpenEdition}

Journals

Édition électronique

URL : http://journals.openedition.org/gradhiva/1774

DOI : 10.4000/gradhiva. 1774

ISSN : 1760-849X

Éditeur

Musée du quai Branly Jacques Chirac

Édition imprimée

Date de publication : 19 mai 2010

Pagination : 225-229

ISBN : 978-2-35744-025-8

ISSN : 0764-8928

Référence électronique

Nathalie Heinich, "Brigitte Derlon et Monique Jeudy-Ballini, La Passion de l'art primitif. Enquête sur les collectionneurs », Gradhiva [En ligne], 11 | 2010, mis en ligne le 09 mai 2010, consulté le 21 septembre 2020. URL : http://journals.openedition.org/gradhiva/1774 ; DOI : https://doi.org/10.4000/gradhiva. 1774

Ce document a été généré automatiquement le 21 septembre 2020.

(C) musée du quai Branly 


\section{Brigitte Derlon et Monique Jeudy- Ballini, La Passion de l'art primitif. Enquête sur les collectionneurs}

Paris, Gallimard, coll. « Bibliothèque des sciences humaines », 2008, $322 \mathrm{p}$.

\section{Nathalie Heinich}

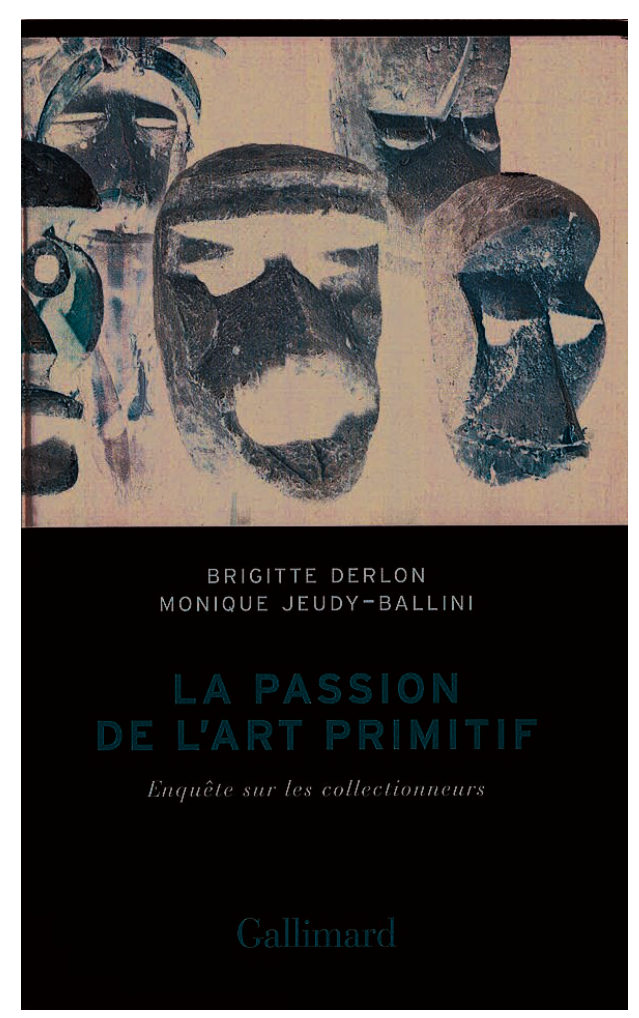

1 En enquêtant sur les collectionneurs d'art primitif dans le monde occidental, Brigitte Derlon et Monique Jeudy-Ballini ont délibérément tourné le dos aux objets traditionnels de leur recherche - les cultures non occidentales - pour porter leurs 
regards vers ceux qui regardent ces objets : «objets » étant à prendre alors au double sens du terme, comme la visée de l'investigation autant que comme la chose matérielle, ce qu'on appelle l'« objet d'art primitif» (ou "premier», "tribal», etc.). Bref, d'ethnologues, elles se sont muées en sociologues. Or, ce faisant, elles ont su importer dans leur entreprise le meilleur de ce que les ethnologues ont à apprendre aux sociologues.

\section{Une perspective compréhensive}

2 En effet, la perspective adoptée dans cette enquête par entretiens est, clairement, la perspective dite «compréhensive» en sociologie, selon l'ancienne opposition expliquer-comprendre mise en évidence il y a un siècle et demi par Wilhelm Dilthey et dont les sciences de l'homme n'ont probablement pas fini d'épuiser la pertinence. Cela signifie deux choses. Premièrement, les auteurs n'ont pas cherché à expliquer la pratique de la collection d'art primitif par des causes extérieures, du type origine sociale, niveau d'études, rapport entre patrimoine et revenus ou encore mobilité géographique, place dans la fratrie, généalogie familiale. Non que ces paramètres, familiers à la sociologie (ou à la psychosociologie) standard, soient sans pertinence ; simplement, ils permettent de dégager les causes générales d'une pratique, mais pas d'expliciter ses raisons, autrement dit le sens qu'elle revêt pour les acteurs ainsi que les conditions précises auxquelles elle est soumise, les critères de choix, les modalités concrètes de rapport aux objets, l'évolution des goûts. Or ce sont justement ces facteurs-là que vise ici l'enquête.

3 La seconde conséquence de ce choix de perspective est que la posture des auteurs n'est, à aucun moment, critique par rapport aux acteurs interrogés. Cela est délibéré : « Tout en reconnaissant les apports du postmodernisme et du postcolonialisme, nous pensons à notre tour qu'il est temps de dépasser la perspective critique pour s'intéresser aux motivations exprimées par les collectionneurs. Fondé sur un parti pris spécifiquement ethnographique, cet ouvrage vise à rendre compte de leurs représentations", précisent-elles dans l'introduction (p.22). Il va donc être question dans ce livre, successivement, du rôle de l'émotion, du hasard, du dépaysement et, enfin, de l'argent.

4 L'argent, justement: que la visée critique soit incompatible avec la perspective compréhensive devient parfaitement évident dès lors qu'il est question de l'argent, thème à propos duquel le réductionnisme critique se trouve toujours à son aise, même s'il mobilise la motivation non plus par l'intérêt matériel mais par le prestige lorsque la collection est interprétée comme "signe extérieur de richesse ». Remarquant que «ce point de vue est rarement partagé par nos interlocuteurs, du moins quand ils parlent d'eux-mêmes ", les auteurs précisent : "Le rapport des collectionneurs à l'argent nous a intéressés, mais pas comme cette vérité dernière et incontournable de la collection qu'en fait parfois le sens commun » (et, pourrait-on ajouter, les chercheurs eux-mêmes, car il semble que les anthropologues ne se privent pas, à l'occasion, d'être aussi critiques que les plus critiques des sociologues critiques lorsqu'il est question de ces frères ennemis que sont les collectionneurs). « Au lieu de l'opposer au fantasmatique et à l'affectif pour y voir la réalité sociale sous-jacente à leur pratique ou encore sa dimension concrète par excellence, nous l'avons appréhendé sous l'angle des représentations qui le soutiennent et en tant que partie prenante du rapport des collectionneurs à l'objet. » (p. 273) 
5 Ainsi débarrassée de toute pulsion critique, cette perspective permet de mettre en évidence la logique sous-jacente à la pratique de la collection, ce qui en elle fait sens pour les acteurs: "Pour nos interlocuteurs, l'objet est clairement un ailleurs: géographique, temporel, mental. Il est ce par quoi l'esprit s'échappe mais aussi, et indissociablement, cela même qui lui échappe. Il se confronte à ce qui transcende l'expérience ordinaire, donne à sentir ou pressentir l'existence de réalités supérieures, se fait porte ouverte sur le mystère, stimule l'imaginaire.»(p.59) Fadaises, lieux communs, reprise naïve des justifications indigènes, rétorqueront sans doute d'une même voix le sociologue-critique et l'anthropologue-à-qui-on-ne-la-fait-pas ? Certes, on pourrait reprocher aux auteurs - toutes proportions gardées - ce que Claude LéviStrauss reprochait à Marcel Mauss dans sa célèbre introduction de 1950 à l'Essai sur le don: le hau invoqué par les indigènes ne peut être posé par l'ethnologue comme une catégorie explicative car la cause de l'échange ne réside pas dans l'objet lui-même, mais dans les structures anthropologiques sous-jacentes aux pratiques de circulation des objets. Or, ce que n'a pas vu Lévi-Strauss, c'est que Mauss ne prétendait pas "expliquer» ainsi, mais «comprendre », c'est-à-dire restituer la logique des représentations qui, pour les acteurs, donnent sens au don. L'une et l'autre perspectives sont d'ailleurs également honorables et, en outre, parfaitement compatibles : c'est affaire de regard, de visée épistémique. Simplement, ce qu'elles rendent intelligible n'est pas du même ordre. Et si l'« explication » que propose LéviStrauss (par les règles de la vie sociale) est incompatible avec celle que produisent spontanément les acteurs (par une propriété constitutive de l'objet donné), en revanche la «compréhension» que propose Mauss (par la représentation partagée d'une force objectivée) est parfaitement compatible avec l'explication donnée par les acteurs, puisqu'elle s'appuie sur elle; mais elle n'en constitue pas une reprise littérale car elle lui ajoute une dimension beaucoup plus générale : celle qui est commune à tous les objets qui circulent comme à tous les sujets qui les font circuler, à savoir les cadres mentaux structurant la place accordée à ces objets et aux pratiques qui leur sont associées.

6 Ici, l'équivalent du hau semble bien être le «beau », cette propriété invoquée par les collectionneurs pour expliquer ce qui les pousse à pratiquer ce type particulier d'échange qu'est la collection. Nos auteurs ne le disent pas explicitement, mais il suffit pour s'en convaincre de remplacer dans la phrase suivante (à propos de la prévalence de l'émotion sur l'esthétique) «beau » par hau: «Le jugement esthétique se fonde moins sur des critères strictement formels que sur des affects fortement liés à l'imaginaire. Ce n'est pas parce qu'il est beau que l'objet fait rêver ou voir le monde autrement; c'est parce qu'il fait rêver ou voir le monde autrement qu'il est beau. Le beau ne constitue pas tant une qualité intrinsèque à l'objet qu'un produit de son interaction avec la personne. L'objet lui procure un transport émotionnel et reçoit d'elle, en retour, la reconnaissance de sa beauté.» (p. 82)

7 Il en va de même avec les capacités d'incitation à l'action que possède - toujours aux yeux des acteurs - l'objet de collection lorsqu'ils le dotent de la faculté de faire agir : "Si l'objet procure en lui-même une sorte d'élévation comme l'attestent les transports de l'imaginaire et les interrogations existentielles suscités par sa présence, il se voit encore reconnaître la faculté de pousser son détenteur à l'action par l'énergie qu'il lui communique. Pour dire bref, l'objet fait tout à la fois rêver, réfléchir et agir. » (p. 87) 
8 La perspective adoptée permet donc de faire apparaître ce que l'objet fait au collectionneur. Et que fait la collection d'art primitif, ainsi explorée, à l'ethnologue? Elle l'incite à tourner un regard "compréhensif ", non critique, sur la logique sousjacente aux pratiques et aux représentations propres à notre culture occidentale : un type de regard que j'avais assimilé, dans mon travail sur Vincent van Gogh, à l'approche anthropologique, mais qu'une pratique plus expérimentée de la sociologie me permet aujourd'hui d'associer aussi à cette discipline. Il s'agit ce faisant, selon le proverbe chinois souvent cité, de regarder non pas la lune (l'objet d'art primitif) que le sage (le collectionneur) montre à l'idiot (l'ethnologue), mais le doigt qui la montre (le regard du collectionneur) - le plus idiot des deux n'étant alors pas forcément celui qu'on croit...

Certes, une telle posture de recherche laisse forcément dans l'ombre certains éléments, que vise la traditionnelle perspective explicative - celle qui continue de regarder la lune, en cherchant à aller plus loin que les acteurs dans la tentative pour en faire le tour. Ainsi, dans ce livre, le lecteur restera sur sa faim quant à la question de savoir ce qui incite à collectionner l'art primitif plutôt qu'à, simplement, s'y intéresser (comme le font les ethnologues) ; ou encore, symétriquement, quant à la question de savoir ce qui incite à collectionner de l'art primitif plutôt que n'importe quels autres types d'objets (la seule indication donnée par les auteurs renvoie à la variable sexe: «La distinction pertinente en la matière est celle du sexe, certains objets étant préférentiellement collectionnés par des hommes - antiquités, voitures, livres, armes, timbres, etc. - et d'autres par les femmes - figurines animales, bijoux, éléments de décoration intérieure, etc. »[p. 28]).

En permettant d'entrer dans les représentations des acteurs plutôt que d'en sortir aussi vite que possible pour leur trouver des causes, cette perspective compréhensive donne tout loisir de déployer les soubassements axiologiques, les cadres cognitifs, les ressources interprétatives à l'œuvre dans le rapport de ces collectionneurs à leurs objets. Ce sont ainsi d'autres directions de recherche qui s'ouvrent, encore peu frayées par nos disciplines. Je me contenterai ici d'en indiquer trois, qui me sont chères et auraient pu, me semble-t-il, être davantage creusées par les auteurs : la question de l'inspiration, la question de la singularité, la question de l'authenticité.

\section{L'état inspiré}

11 J'ai eu pour ma part la surprise de retrouver en lisant ce livre, dans ce monde qui m'était inconnu, des caractéristiques que j'avais rencontrées dans le rapport entretenu par les écrivains avec la création. Elles relèvent de ce qu'on associe dans notre culture avec la "mystique » ou la "spiritualité », et que la sociologie critique a tôt fait de réduire au statut d'« illusion » avant même de se demander ce qu'elles signifient pour les acteurs, dans quelles configurations elles apparaissent, à quels types d'expériences elles sont associées. Ici, on les rencontre sous deux formes typiques de la création en « régime de singularité » : l'énigme et la passivité.

12 Il existe en effet chez les acteurs interrogés une propension à postuler un " mystère » inhérent à l'objet - ce que j'ai nommé ailleurs une "mise en énigme », indissociable chez nous de la mise en valeur de certaines entités, de leur érection au rang d'objets d'admiration. Brigitte Derlon et Monique Jeudy-Ballini le relèvent à propos des collectionneurs, qui « assimilent le sacré - entendu dans une acception non religieuse à ce qu'ils pressentent chargé de significations plus ou moins obscures, porteuses d'une 
dimension mythique, spirituelle ou métaphysique, les renvoyant à des interrogations existentielles sur leur rapport au monde » (p. 80).

En outre, l'état de collectionneur est souvent décrit comme un état de passivité, tout à fait analogue à celui dont font état les écrivains à propos de l'inspiration : un état propice à l'envahissement par une force extérieure, transcendante, qui fait de l'écrivain - ou, ici, du collectionneur - le simple instrument, le médiateur entre cette force et les objets ou les œuvres en lesquels elle vient s'incarner. Alors, c'est l'objet collectionné (ou le roman, le poème, etc.) qui devient le véritable acteur, le détenteur de la force, et le collectionneur (ou l'auteur) qui devient son " sujet ", au sens de celui qui obéit et qui sert : «Si le collectionneur a bien pris la décision de se déplacer dans un pays étranger, de circuler dans les rues de la ville, de s'arrêter devant un immeuble inconnu, de gravir des escaliers en frappant à toutes les portes, ce n'est malgré tout pas lui qui est allé à la rencontre de l'objet, mais l'objet qui a fait en somme une trouvaille en la personne du collectionneur. Au lieu de mettre en avant la persévérance nécessaire pour obtenir gain de cause, ce dernier tend à s'effacer, comme si comptaient pour rien ses intentions et ses efforts. L'initiative est du côté de l'objet qui "sort", "tombe", lui est "donné", son futur détenteur semblant avoir été élu, choisi par lui et non l'inverse. À l'instar des populations dont les mythes attribuent une origine non humaine aux effigies rituelles fabriquées par les hommes (et futures pièces de collection!), les collectionneurs occultent la part active prise par eux dans la découverte de l'objet; un objet dont l'apparition "inattendue" évince la dimension rationnelle de la trouvaille et n'est pas loin de s'apparenter à une sorte de mythe fondateur. » (p.117)

14 Ici, des comparaisons avec l'expérience mystique, telle qu'elle a pu être décrite ou étudiée dans d'autres domaines, auraient sans doute permis d'éclairer et de généraliser le propos.

\section{Les objets-personnes}

15 Une autre dimension récurrente dans le rapport à ce type de collection est le statut de singularité conféré aux objets en question, au double sens où ils sont uniques, particuliers, insubstituables, et où ils sont en même temps bizarres, étranges, hors du commun. Ils sont donc parfaitement représentatifs de la catégorie de ce que j'ai nommé les « objets-personnes », définis par leur insubstituabilité.

C'est ainsi que, telles des personnes humaines, ils apparaissent dotés d'« autonomie », d'« interaction » et de « réciprocité » : « Maintes fois illustrée par les œuvres littéraires, dont celles de Balzac et de Maupassant déjà évoquées, l'assimilation de l'objet à une personne est un procédé récurrent chez les collectionneurs qui rend compte de divers aspects de la relation que les hommes entretiennent avec les choses. La façon dont sont vécus l'attirance et l'attachement pour l'objet (à la fois résultat d'une attraction soudaine et forme de communication) tient un rôle essentiel dans cette assimilation qui se joue notamment autour des notions d'autonomie, d'interaction et de réciprocité. » (p. 64) Grâce à l'émotion qu'il suscite, l'objet d'art primitif devient « davantage qu'une chose: une réalité inépuisable, une présence, une entité souvent créditée de formes d'action ou d'intentionnalité. Cette autonomie prêtée à l'objet se fonde sur l'impression récurrente des collectionneurs d'avoir été élus par lui davantage qu'ils ne l'ont choisi. " (p. 156) La métamorphose de la chose en personne va même jusqu'à s'inverser avec la transmutation rêvée du collectionneur en objet: au devenir-personne de l'objet fait 
pendant, symétriquement, le devenir-chose de la personne lorsque, dans les représentations, «l'objet s'autonomise jusqu'à acquérir un statut de quasi-personne en même temps que son détenteur tend à perdre son autonomie jusqu'à se penser ou se rêver lui-même comme pièce de collection » (p. 201).

L'analyse toutefois aurait gagné à se prolonger en prenant en compte les différentes catégories d'« objets-personnes » : le fétiche, agissant comme une personne ; la relique, ayant appartenu à une personne; et l'œuvre d'art, traitée comme une personne humaine. L'objet d'art primitif, en effet, semble particulièrement à même de basculer dans ces différents états selon qu'on l'envisage dans son contexte ethnologique, dans sa « carrière » d'objet ou dans le contexte de la collection.

\section{La question de l'authenticité}

Une troisième et dernière problématique qu'abordent les auteurs, mais sans lui donner peut-être toute l'attention qu'elle mériterait, est celle de l'authenticité, en tant qu'attestation d'un lien avec l'origine, quelle qu'elle soit. Car dans le cas des objets d'art primitif, là encore, cette question est particulièrement aiguë du fait que l'origine n'est pas seulement géographique et temporelle, comme dans le cas des objets d'art occidentaux, mais aussi fonctionnelle: il faut absolument que, comme on dit, «le masque ait dansé ", ou en tout cas qu'il n'ait pas été créé pour la seule délectation de l'amateur étranger.

Les auteurs le soulignent: "Certains types de collections s'accommodent parfaitement du caractère neuf ou non utilisé des objets. Pas celle d'art primitif. La massue ne sert certes plus à assommer, le couteau de circoncision à couper, l'amulette à protéger ; mais ils doivent avoir effectivement assommé, coupé et protégé dans leur société d'origine. Selon une formule usitée dans le milieu des collectionneurs et marchands : "Il faut que le masque ait dansé." L'objet ne devient collectionnable et doté de valeur reconnue qu'en tant qu'il est supposé avoir servi.» (p. 47) Ainsi augmentée de ce que certains collectionneurs appellent la "patine d'usage", l'authenticité ne rend pas seulement l'objet plus « cher » (dans tous les sens du terme) mais aussi, pour ainsi dire, plus réel : «En somme, ce n'est pas parce qu'il existe que l'objet serait "réel". Pour être confirmée, sa "réalité" nécessite une sorte de procédure d'authentification. Celle-ci réside en l'occurrence dans le constat de l'usage effectif de l'objet à travers les traces laissées à sa surface (un "tampon de vérité"). » (p. 49)

Sans doute est-ce dans cette direction qu'il faudrait poursuivre pour mettre en évidence la spécificité de l'art primitif par rapport à d'autres types de collections : non pas tant la mise en énigme (que l'on trouve aussi à propos des objets d'art occidentaux), mais plutôt la dimension d'étrangeté, d'éloignement, d'exotisme, qui rend encore plus fragile, donc plus précieux et porteur d'émotion, le sentiment vécu du lien avec son origine; ainsi que - probablement- la rusticité de ces objets, leur relatif dépouillement, l'économie de leurs matériaux ou de leur iconographie, qui rendent encore plus sensible, par contraste, ce qui reste en eux d'essentiel -à savoir la continuité du lien avec le lieu et le moment où ils furent créés, et qu'on nomme, chez nous, « authenticité ». 
21 Reste donc à souhaiter que d'autres "enquêtes au pays des collectionneurs » prolongent celles-ci dans la direction prise ici par Brigitte Derlon et Monique JeudyBallini, de façon à approfondir les nombreuses pistes qu'elles ont su dégager.

\section{AUTEUR}

NATHALIE HEINICH

heinich@ehess.fr 